\title{
Os jornalistas em Porto Rico frente os fundamentos de sua profissão no século XXI
}

\author{
Los periodistas en Puerto Rico ante los fundamentos de su profesión en el siglo XXI \\ Journalists in Puerto Rico forward the foundations of his profession in the XXI \\ century
}

Lourdes Lugo-Ortiz

(lourdeslugoortiz@gmail.com $)^{1}$

http://dx.doi.org/10.5216/cei.v15i1.22502

\begin{abstract}
Resumo
Qual é a percepção dos jornalistas sobre os valores e as funções da sua profissão? O interesse gerado por essa questão está ancorada na idéia de que aparência profissional pode afetar o conteúdo da informação que produz. $\mathrm{Na}$ América Latina os estudos com foco na figura do jornalista começaram a proliferar a partir da década de noventa, apesar de serem uma minoria aqueles papéis profissionais (Mellado, 2010). Este ensaio, portanto, pretende investigar, através do método da pesquisa, que, de acordo com jornalistas na ilha, são os valores fundamentais da profissão, em um período em que a entrada da Internet tem impactado a forma do jornalismo e colocou em crise a estabilidade econômica e a hegemonia de reportagens da mídia comercial. Para explorar este fim, este estudo adotou o projeto da pesquisa realizada pelo Pew Research Center for the People e da imprensa.
\end{abstract}

Palavras-chave: Jornalistas. Porto Rico. Fundamentos Profissionais.

\section{Resumen}

Cuál es la percepción que tienen los periodistas sobre los valores y roles de su profesión? El interés que genera esta pregunta se ancla en la idea de que la mirada del profesional de la información puede incidir en el contenido que produce. En Latinoamérica los estudios centrados en la figura del periodista comenzaron a proliferar a partir de la década de los noventa, aunque se encuentran en minoría aquellos sobre los roles profesionales (MELLADO, 2010). Este ensayo, entonces, tiene el propósito de indagar, mediante el método de la encuesta, cuáles, de acuerdo con los periodistas en la Isla, son los valores fundamentales de la profesión, en un periodo en que la entrada de la Internet ha impactado la forma de hacer periodismo y ha puesto en crisis la estabilidad económica y la hegemonía informativa de los medios comerciales. Para explorar este objetivo, este estudio adoptó el diseño de la encuesta realizada por Pew Research Center for the People and the Press.

Palabras-clave: Periodistas. Puerto Rico. Fundamentos Profissionales.

\begin{abstract}
What is the perception of journalists on the values and roles of their profession? The interest generated by this

1 Ph.D., Catedrática - Escuela de Comunicación, Universidad de Puerto Rico, Río Piedras, Puerto Rico.
\end{abstract}


question is anchored in the idea that professional look can affect the information content it produces. In Latin America, studies focusing on the figure of the journalist began to proliferate from the nineties, but those are in the minority on professional roles (MELLADO, 2010). This paper, then, aims to investigate, using the method of the survey, which, according to reporters on the island, are the core values of the profession, in a period in which the entry of the Internet has impacted the form of journalism in crisis and has put economic stability and informational hegemony of commercial media. To explore this objective, this study adopted the design of the survey conducted by Pew Research Center for the People and the Press.

Keywords: Journalists. Puerto Rico. Fundamentals Professionals.

\section{Introducción}

¿Cuál es la percepción que tienen los periodistas sobre los valores y roles de su profesión? El interés que genera esta pregunta se ancla en la idea de que la mirada del profesional de la información puede incidir en el contenido que produce. No es de extrañar, entonces, que, en el campo de la Comunicación, este tema haya sido objeto de estudio sostenido desde la década de los setenta (JOHNSTONE, SLAWSKI Y BOWMAN, 1976; WEAVER y WILHOIT, 1986 y 1996; WEAVER et al., 2007, y BEAM, WEAVER y BROWNLEE, 2009).

La mayor cantidad de investigaciones sobre el papel social de los periodistas se ha elaborado en Estados Unidos, Canadá y Europa (HOYER y LAUK, 2003 y PRITCHARD y BERNIER, 2010). En Latinoamérica, por su parte, los estudios centrados en la figura del periodista comenzaron a proliferar a partir de la década de los noventa, aunque se encuentran en minoría aquellos sobre los roles profesionales (MELLADO, 2010). Pese a la importancia de este tipo de análisis, en Puerto Rico, dicho campo ha sido inexplorado. Este ensayo, por tanto, tiene el propósito de indagar, mediante el método de la encuesta, cuáles, de acuerdo con los periodistas en la Isla, son los valores fundamentales de la profesión, en un periodo en que la entrada de la Internet ha impactado la forma de hacer periodismo y ha puesto en crisis la estabilidad económica y la hegemonía informativa de los medios comerciales.

\section{La Comisión Hutchins}

El debate sobre el rol del periodismo en una sociedad democrática tiene su génesis a principios del siglo XX en los Estados Unidos y en Europa, y, con ello, la discusión acerca de su profesionalización, aunque algunos académicos aseguran que el proceso se inició a mediados del siglo XIX (BANNING, 1998-99; HALLIN, 2000, p. 219; HARCUP, 2002, p. 101; BIRKHEAD, 2003 y 
HOYER y LAUK, 2003, p. 3). Liderado en parte por The New York Times y mimetizado luego por otros periódicos, ya durante las primeras décadas del siglo XX en los Estados Unidos, se había consolidado el llamado a la profesionalización del periodismo, o a una ética de "servicio público" en función de la ciudadanía y apegada al valor de la objetividad (HALLIN, 2000, p.219-220). En Puerto Rico, tal convocatoria se acogió con la fundación del periódico El Mundo en 1919, cuando se comenzó a establecer con fuerza la práctica de un periodismo alejado de las alianzas político-partidistas y con intereses comerciales. $^{2}$

Haciendo eco a las demandas articuladas a principios del siglo XX, la Comisión Hutchins sobre la Libertad de Prensa en los Estados Unidos preparó, en 1947, un informe relativo al papel social del periodismo. El reporte surge de la preocupación que presenta el aumento en la concentración de los medios, su extrema comercialización y que los esfuerzos para impedir la interferencia del gobierno en el quehacer periodístico no fueran suficientes para proteger el interés público. Ante este panorama, la inquietud de la Comisión giraba en torno a dos asuntos principales: que los dueños de los medios opacaran las perspectivas políticas que fueran contrarias a las de ellos y que la extrema comercialización de la prensa torpedeara la producción de contenido responsable. El informe aboga por que el gobierno tome unas acciones particulares para promover la competencia y por que las organizaciones sin fines de lucro suplementen el trabajo de los medios. Mas, lo central del texto es que hace un llamado a la profesionalización del periodismo, arraigado en que la lealtad principal de su trabajo sea el interés ciudadano y no intereses particulares. Con ese propósito, el reporte enumera cinco responsabilidades de la prensa en una sociedad democrática: presentar una relación verídica, completa e inteligente de los acontecimientos diarios en un contexto que les dé significación; servir de foro para el intercambio de comentarios y crítica, presentar una imagen representativa de los grupos que constituyen la sociedad, explicar las metas y valores de la sociedad y garantizar el acceso pleno a la información relevante del acontecer diario (Commission on the Freedom of the Press, 1947).

El informe, luego, sirvió de base para articular la "Teoría de la responsabilidad social de la Prensa", de Siebert, Peterson y Schramm (1956), la que, sin lugar a dudas, ha tenido una fuerte influencia en las formas en que se ha concebido el rol de la prensa en una sociedad democrática en los Estados Unidos, en Europa y en el mundo entero, incluyendo a Puerto Rico (En WEAVER y

2 Véase a Coss (2007) para la historia de la profesionalización del periodismo en Puerto Rico. 
WILHOIT, 1986, p. 9-11 y en HALLIN, 2000< p. 219). Precisamente, respondiendo a esa línea teórica, se ha articulado que la razón de ser del periodismo es "proporcionar a los ciudadanos la información que necesitan para ser libres y capaces de gobernarse a sí mismos” (KOVACH y ROSENSTIEL, 2003, p. 26).

Según Weaver Wilhoit (1986, p.10), las perspectivas de la Comisión sobre la responsabilidad social del periodismo han sido generalmente aceptadas por la prensa y la sociedad en general; no obstante, estas han entrado en contradicciones y en tensión con los medios de comunicación, el gobierno y otras instituciones. Esa resistencia impulsó a explorar, a partir de la década de los setenta y de forma consecuente, cómo los miembros de la prensa perciben su papel profesional.

\section{Otros estudios importantes}

La percepción que los periodistas tienen sobre su rol social cuenta con un sólido caudal investigativo que se inicia en los Estados Unidos en la década de los setenta, aunque desde la década de los treinta comienzan a publicarse estudios de menor envergadura (JOHNSTONE, SLAWSKI y BOWMAN, 1976, p. 2-4). En medio de los disturbios provocados por la guerra de Vietnam, surge, en 1971, la primera encuesta realizada a todos los periodistas en los Estados Unidos. En esta, Johnstone, Slawski y Bowman (1976) identificaron dos conjuntos de creencias que tenían los periodistas sobre su rol social: el periodista "neutral", como observador objetivo de la realidad, cuya función primaria es informar a su público, y el periodista "participante", aquel que se percibe más activo, que interpreta, explica y, quizás, presenta soluciones a los problemas sociales (1976, p.113-132). El primero se adhiere a los valores de la objetividad, precisión y verificación de la información, y el segundo, a los valores de relevancia, contexto, análisis e interpretación (JOHNSTONE SLAWSKI y BOWMAN, 1976, p. 114115).

A partir de la década de los ochenta y en varias investigaciones, Weaver y otros académicos retomaron el estudio de Johnstone et al., y ampliaron el marco de análisis original. En el primer estudio —en 1982-1983, ya cuando el reportaje investigativo en los Estados Unidos había hecho su agosto con el caso Watergate-, Weaver y Wilhoit (1986, p. 115), nombraron "difusor" al periodista "neutral". Esta redefinición implica que el papel de informador no necesariamente es neutral o no participativo, sino el apego a los datos y a la verdad es lo que se constituye en elemento central. Al "participante", 
por otro lado, lo llamaron "interpretador", distanciándose de la noción de que el contexto y la investigación son sinónimos de participar en la información. Asimismo, le añadieron un tercer rol al modelo: el de "adversario" del poder económico o gubernamental.

En los estudios de Johnstone et al. (1976), de Weaver y Wilhoit (1986 y 1996), Weaver et al. (2007) y Beam et al. (2009), se evidenció que los periodistas en los Estados Unidos ven su rol de forma pluralista: se perciben tanto como difusores e intérpretes, $\mathrm{y}$, de forma minoritaria, como adversarios del poder. Sin embargo, en el estudio de Weaver y Wilhoit en la década de los noventa, emergió una cuarta función, también identificada de forma minoritaria: la del periodista "activista-populista" (populist mobilizer), que promueve el desarrollo de los intereses del público, provee entretenimiento y establece la agenda política de la audiencia (WEAVER y WILHOIT, 1996, p. 140).

Aunque ven su rol de forma pluralista, los periodistas en los estudios de Weaver, Wilhoit y otros han identificado el papel de interpretador como el más importante (WEAVER y WILHOIT, 1986 y 1996; WEAVER et al., 2007, y BEAM et al., 2009). No obstante, en la investigación del 2002, aumentó la percepción del que combina el rol de "interpretador" con el "de activista-populista" (2007, p. 146) y, en la realizada entre el 2002 y el 2007, incrementó el que combina el periodista "interpretador" con el de "adversario" del poder (BEAM et al., 2009). Los autores atribuyen este cambio a los retos económicos y a la pérdida de audiencia que la Internet les ha presentado a los medios tradicionales

Communication technologies like the Web were reshaping the way journalists do their work. Coincident with these technological challenges - or perhaps because of them - news organizations were facing one of the most hostile business environments in decades. All these changes had the potential to influence journalists' views about their professional roles. (BEAM et al., 2009, p. 285 y WEAVER et al., 2007, p. 143).

La irrupción del periodismo cibernético y la proliferación de las colaboraciones ciudadanas (lo que algunos llaman periodismo ciudadano) hacen más necesario que los periodistas asuman el rol de interpretador, o al menos se piensen como uno. Dice Singer: "As the explosion of information continues, there will be an increasing need for skilled journalists to sort through it, filter out what's important and help put in perspective” (En WEAVER et al., 2007, p. 142). La pregunta que se formulan Beaver et al. es si es posible asumir el rol de interpretador-adversario al interior de las 
empresas periodísticas tradicionales, en medio de un periodo de crisis económica que se caracteriza por cierres, eliminación de plazas y reducción de ingresos publicitarios, particularmente para la prensa escrita (2009, p. 291).

Al corpus investigativo antes mencionado, se le une una importante encuesta realizada, en 1999, por el Pew Research Center for the People and the Press titulada: "Striking the Balance, Audience Interests, Business Pressures and Journalists", cuyo objetivo era precisar cuáles son los elementos esenciales que caracterizan al periodismo de acuerdo con los periodistas estadounidenses. Kovach y Rosenstiel plasmaron los resultados de dicha pesquisa en su libro Los elementos del periodismo (2003), en el que enumeran nueve principios que definen la profesión, los cuales, sin lugar a dudas, conversan con las responsabilidades del periodista planteadas por la Comisión Hutchins cincuenta años antes y con los estudios de Johnstone et al., Weaver y otros. Explican que la obligación del periodista es con la verdad, que su lealtad principal es hacia ciudadanía y que en su trabajo debe primar la disciplina de la verificación. El periodista, de la misma forma, debe mantener independencia de quienes informa, ejercer un trabajo desapegado del poder (ambos para evitar que la información que produzca se vea comprometida) y proveer un foro público para la crítica y el debate. Además, debe esforzarse por que el contenido sea sugerente y relevante, por lo que las noticias deben ser exhaustivas y proporcionadas (de acuerdo con su importancia y a base de la evidencia recopilada). Asimismo, la profesión debe respetar la conciencia individual de sus profesionales, por lo que el periodista tiene la obligación de disentir o enfrentarse al poder, incluyendo a su propia empresa, anunciantes o ciudadanos "si la verdad y la equidad lo exigen” (KOVACH y ROSENSTIEL, 2003, p. 26, 250 y 254).

Las investigaciones anteriormente presentadas han estimulado la producción empírica sobre los periodistas alrededor del mundo (WEAVER, 1998, y REESE, 2001, p. 179). Incluso, varios estudios han encontrado que el modelo profesional periodístico — que nació en los Estados Unidos y en Gran Bretaña y en el que se ve el rol del periodista como informador imparcial, que distingue los hechos de la opinión y que influye en los gobernantes, entre otros elementos - se ha replicado en otros países con contextos nacionales distintos, incluidos los países Latinoamericanos (MANCINI, 1999, p. 132 y MELLADO, 2009). Según Mancini, esta primacía se debe a que este prototipo es el que se ha recogido en los libros, se discute en las universidades y es el que se ha adoptado en los códigos de ética en diferentes países. Señala, por eso, que el paradigma norteamericano "ha ejercido (y todavía ejerce) una 
hegemonía indiscutible. Hasta el punto de que no parece fuera de lugar hablar de un 'lugar común' del modelo profesional" (MANCINI, 1999, p. 132). ${ }^{3}$

\section{Roles, organizaciones y extrema comercialización}

Si bien la exploración de los roles del periodismo se ha centrado en el periodista como sujeto, Shoemaker y Reese (1996) distinguen cinco niveles de análisis para precisar la influencia a la que es susceptible el contenido de los medios: el nivel individual, el de las rutinas de la profesión, el organizativo, el externo a los medios y el ideológico. Sin embargo, varios estudios han identificado que el nivel organizativo es el que tiene mayor impacto en la percepción de los roles profesionales de los periodistas. Estos actúan en simbiosis con sus organizaciones; en términos generales, deben adaptarse a las condiciones de sus medios (JOHNSTONE, SLAWSKI y BOWMAN, 1976; BEAM, 1988; HALLIN, 2000; HOYER y LAUK, 2003; BIRKHEAD, 2003, p. 117, y PRITCHARD y BERNIER, 2010). Es decir, son las condiciones organizativas o el ambiente de trabajo los que predicen la orientación periodística más que los valores individuales, profesionales o factores externos (WEAVER y WILHOIT, 1986, p. 117-119, 178; WEAVER y WILHOIT, 1996, y WEAVER et al., 2007, p. 147). Pese a los hallazgos de Weaver y otros, Hallin (2000, p. 221) indica que solo es posible entender los valores profesionales si se examina tanto el contexto económico organizativo en el que trabajan los periodistas como el conjunto de factores políticos y culturales que han modificado las condiciones en las que floreció el modelo profesional del periodismo.

Dentro de la economía organizativa, y relacionado con lo que Weaver y otros plantean, la excesiva comercialización de los medios impacta el contenido periodístico y las rutinas de producir la noticia (HALLIN, 2000). No se debe olvidar que los valores fundamentales de la profesión fueron desarrollados al interior de una prensa moderna y comercial desarrollada en el siglo XX, que dejaba atrás el modelo del periodismo político. En este tipo de estructura, las fuerzas del mercado inciden en el producto informativo; se espera que este sea rentable. Publicidad y ganancias han sido parte del modelo

\footnotetext{
${ }^{3}$ En un estudio comparativo entre 21 naciones, Weaver (1998 y 1999) halló que las influencias sociales, especialmente las diferencias o similitudes políticas entre los países en los que los periodistas laboran, influyen en las percepciones que tienen sobre su rol más que las organizaciones, la educación en periodismo y las normas profesionales. Precisamente, Hoyer y Lauk (2003, p. 9) enumeran una gran cantidad de estudios.
}

Comum. \& Inf., v. 15, n.1, p. 105-131, jan./jun. 2012 
económico de los medios en los Estados Unidos, Europa y otros países, incluyendo a Puerto Rico, e impactan las maneras de hacer periodismo (BAGDIKIAN, 1983; HERMAN y CHOMSKY, 1988, p. 25; SHOEMAKER y REESE, 1996, p. 146-173; HOYER, SVENNIK y LAUK, 2003, p. 3, BIRKHEAD, 2003, p. 123, y MCCHESNEY, 2003, p. 299-301). A la luz de este modelo, es que se identifican el creciente sensacionalismo y la banalización de los contenidos informativos, práctica que se inició entre finales del siglo XIX y principios del siglo XX (MCCHESNEY, 2003, p. 301) y que en 1947 fue identificada con toda precisión por la Comisión Hutchins (Commission on the Freedom of the Press, 1947, p. 67). En la década de los setenta, resurgió con la competencia que presentó la televisión a los otros medios, y ha cobrado una tercera oleada en los medios tradicionales con la irrupción de Internet. Insertos en ese estilo es que las historias de crímenes, desastres, celebridades y chismes han proliferado en los medios tradicionales y cibernéticos, porque se piensa que atraen a mayores audiencias. Este tipo de periodismo ha afectado de forma transversal a casi todas las plataformas periodísticas. Para Steimberg (2000, p. 235), “en las últimas décadas, se ha hecho cada vez más difícil diferenciar los rasgos textuales de las publicaciones tradicionalmente definidas como amarillas o sensacionalistas de las de aquellas clasificadas como 'serias'”. Según Siegel, el sensacionalismo y la banalización del periodismo del siglo XXI ha alcanzado un nuevo nivel, potenciado por los desarrollos tecnológicos. Afirma que el periodismo de Internet toma en cuenta como único criterio de éxito el índice de "popularidad", ya que el énfasis en el despliegue noticioso se determina a base de la cantidad de usuarios que acceden a la información en determinado tiempo, no por la calidad informativa. El número de visitas define el contenido periodístico a publicar tanto en la plataforma digital como en la tradicional. El problema, de acuerdo con Siegel, es que estos actos equiparan "la información con el poder de conocimiento". Son rutinas que, al fin y al cabo, llevan a desvalorizar el propio conocimiento y lo convierten en simple información (2008, p. 156).

Los periodistas se asientan en un terreno movedizo que afecta la percepción de sus valores profesionales. Por un lado, deben producir contenido para un medio que labora tanto para la ciudadanía como para el mercado

Journalists operate in (at least) two worlds, working in a field that is (ostensibly) constituted by a professional commitment to ethics and truth telling while at the same time being expendable employees expected to produce whatever stories are demanded

Comum. \& Inf., v. 15, n.1, p. 105-131, jan./jun. 2012 
in the market- place.” (O’NEILL y BELSEY EN HARCUP, 2002, p. 103).

Mas, por otro, la forma en que los periodistas perciben los valores no se puede divorciar de las condiciones diarias de su trabajo al interior de su organización: "Understaffing, job insecurity, casualised labour, bullying and unconstrained management prerogative" (HARCUP, 2002, p. 112).

La excesiva comercialización de los medios no solo incide en cómo los periodistas perciben los valores de la profesión, sino también, de acuerdo con Hallin (2000), los cambios políticos culturales que se han dado particularmente a partir de la década de los sesenta y que han impactado el modelo del periodismo. Entre estos, vale mencionar desde las luchas feministas y los derechos civiles hasta la entrada de la Internet, y, con ello, las formas y los vehículos que los públicos usan para acceder y producir información. Según algunos estudios, todos estos factores afectan el rol del periodismo, pero más que nada inciden en la percepción que los periodistas tienen sobre su papel profesional (HALLIN, 2000 y WEAVER et al., 2007).

El periodismo del siglo XXI en Puerto Rico, con una prensa moderna consolidada, vive, al igual que el de muchos otros países, un momento de transición en el que los medios informativos tradicionales se han visto insertos en cambios que afectan su estabilidad económica y producción de contenidos. La proliferación de plataformas informativas en el mundo cibernético ha impactado el negocio de los medios tradicionales. Esto es así pese a que la penetración de la Internet en la Isla solo alcanza para junio de 2011 un 37.3 por ciento, aunque en los últimos años esta cifra ha escalado de forma vertiginosa (World Internet Stats, 2011) y muchos lectores de prensa tradicional han migrado a la Internet. ${ }^{4}$ Asimismo, el uso de las redes sociales como vehículos informativos, la creación de espacios periodísticos alternos (algunos de ellos impulsados por periodistas), el envejecimiento de la población que lee los medios impresos, la falta de un modelo publicitario exitoso para las plataformas cibernéticas, entre otros, son algunos de los desafíos que enfrentan los medios tradicionales (MEYER, 2004; IGARZA, 2008; SALAVERRÍA y NEGREDO, 2008; VALDETTARO, 2009; JONES, 2009, FULLER, 2010 y FRANCO, 2010). El reto económico en la Isla se ha traducido en cierres de medios (The San Juan Star y la mesa de español de Associated Press) y en la reducción y eliminación de

\footnotetext{
${ }^{4}$ Las estadísticas de Internet World Stats se anclan en la cantidad de usuarios de Facebook para dicha fecha. Sin embargo, una investigación realizada por Estudios Técnicos con una muestra probabilística de 500 sujetos indica que, para el 2010, el 45 por ciento de la población puertorriqueña está conectada a la Web. El estudio tiene un margen de error de más o menos 4.48 (Custodio Collazo, 2010, p. 35).
} 
plazas (Canal 6 y 11, Primera Hora, El Nuevo Día y El Vocero, entre otros). Además, los periodistas en la Isla han tenido que amoldar sus rutinas a la creación de contenidos simultáneos tanto para la plataforma tradicional como para la cibernética. Refiriéndose a otros países, algunos estudiosos han señalado que estos cambios han golpeado la moral de los periodistas que laboran en los medios informativos y la forma que perciben cuál es su rol social. Incluso, al referirse a los Estados Unidos, Beam et al. (2009, p. 282) catalogan este periodo como uno incierto para los periodistas de su país

Journalists' professional autonomy has been under assault from many quarters in recent years. The growing commercialization of the news, the high profit expectations $f$ news organizations, the shrinking wall between journalistic and business operations, and the introduction of new media technologies all have been blamed for reducing journalists' professional discretion. Today, the eroding financial health of many news organizations might be added to that list.

En este contexto, este estudio se orienta a explorar cómo se posicionan los periodistas en la Isla ante los valores fundamentales de la profesión.

\section{Método y perfil del encuestado}

Este ensayo se dirige a contestar tres interrogantes: de acuerdo con los periodistas en Puerto Rico, ¿cuáles son los elementos que distinguen el periodismo?, ¿cuáles son los valores fundamentales de la profesión?, ¿existen diferencias en las percepciones cuando se examinan las variables de carácter organizativo - medio en el que laboran y puesto que ocupan - y los años de experiencia? Aunque se reconoce su importancia, está fuera del alcance de este ensayo explorar cómo las percepciones de los periodistas afectan el contenido que producen.

Para contestar las preguntas de investigación, este estudio adoptó el diseño de la encuesta realizada por Pew Research Center for the People and the Press. ${ }^{5}$ Pese a que los estudios de Johnstone et al., Weaver y otros antes citados no constituyen la base del esquema investigativo de esta pesquisa, estos, al igual que los otros reseñados, servirán como marco de referencia para el análisis que aquí se presenta.

La encuesta de 43 preguntas fue realizada del 1 de marzo al 2 de abril de 2010 por correo

\footnotetext{
${ }^{5}<$ http://people-press.org/report/67/striking-the-balance-audience-interests-business-pressures-and-journalists-values>.
} 
electrónico y fue dirigida a los periodistas activos de los trece principales medios informativos de Puerto Rico: cuatro diarios con sus respectivas plataformas cibernéticas (El Nuevo Día, El Vocero, Primera Hora y Puerto Rico Daily Sun), cinco estaciones de radio informativas (WKAQ-AM, Radio Isla, Noti-Uno, Wapa Radio y Boricua 740) y cuatro estaciones de televisión (Canales 2, 4, 6 y 11). Este ensayo se basa solo en algunas de las preguntas del cuestionario. En esta investigación, se definen como "periodistas activos" aquellos que laboran en un medio informativo, ya sea a tiempo parcial o a tiempo completo, y que producen o colaboran en la producción del contenido periodístico informativo, entiéndase: jefes de sección, editores, reporteros, productores, presentadores y fotoperiodistas. Quienes laboran como colaboradores fueron excluidos del estudio.

Para propósitos del muestreo, se le pidió a cada medio nacional que sometiera una lista de todos los profesionales que laboran a tiempo completo o parcial en sus salas de redacción con sus respectivas direcciones electrónicas. De un total de 357 miembros de la prensa, 113 contestaron la encuesta. El margen de error corregido de la encuesta para una población finita (CFP) ${ }^{6}$ oscila por pregunta entre +5.7 y +-8 con un 95 por ciento de nivel de confianza.

La mayoría de los encuestados labora para periódicos (77.7\%), para la versión en línea de algún medio $(44.7 \%)$, y el resto, para televisión $(12.9 \%)$ y radio $(4.7 \%){ }^{7}$ Estos porcentajes coinciden con el escenario laboral de la Isla, en el que los diarios, con su componente digital, absorben una mayor cantidad de empleados que los otros medios.

Los reporteros constituyen el grupo con mayor representación en el estudio (65\%), a los que les siguen los jefes de sección (22.9\%). Seis de los trece directores de los medios también contestaron la encuesta, lo que equivale a un 46 por ciento de ellos. La mayor cantidad de los participantes son aquellos que llevan 13 años o más como periodistas (46.4\%), siguiéndole en orden descendente los que tienen experiencia laboral de ocho a doce años $(25 \%)$, de cuatro a siete años (25\%) y los que llevan tres años o menos en el campo periodístico (3.6\%). Una limitación del estudio gira en torno a la poca representación en la muestra de aquellos con tres años o menos de experiencia o aquellos que fungen como productores-as, fotoperiodistas y laboran en la radio, lo que inhibe que se establezca un análisis diferenciado de estas categorías en los resultados aquí presentados. Se podría delinear, entonces, el

\footnotetext{
${ }^{6}$ Tamaño de la muestra corregido para una población finita $=1+n /(n-1 / p o b$.$) .$

${ }^{7}$ Muchos de los periodistas trabajan en más de un medio informativo; por eso, el porcentaje supera 100.
}

Comum. \& Inf., v. 15, n.1, p. 105-131, jan./jun. 2012 
perfil de los encuestados como un reportero o reportera que trabaja en un rotativo o en Internet con una experiencia laboral de ocho años o más.

A continuación, se presentan los resultados del estudio divididos en tres secciones, seguidos por las conclusiones.

\section{5 ¿Qué distingue al periodismo?}

Cuando se les pidió a los periodistas que describieran en sus propias palabras las características que distinguen al periodismo, quedó claro que no existe una opinión mayoritaria que demuestre que los encuestados coinciden en los atributos específicos que diferencian su profesión. Esto fue así independientemente del puesto que ocupan, años de experiencia o medio en el que trabajan.

Los atributos que distinguen al periodismo citados con mayor frecuencia son: informar (49\%), apego a la verdad y honestidad (36\%) y reportar de forma objetiva, imparcial, justa y balanceada $(28 \%)$. El primero tiene que ver con el servicio que el periodismo le brinda a la ciudadanía, y los otros dos, con los valores que, para ellos, deben imperar en el ejercicio periodístico ${ }^{8}$. Estas dos categorías amplias también cobijan otros atributos mencionados por los encuestados, aunque en porcentajes menores [e.g., interpretar (valor profesional), impacto (servicio a la ciudadanía), etc.].

Los tres atributos mencionados con mayor frecuencia — informar, verdad y objetividaddenotan que muchos de los periodistas en la Isla perciben el rol de "difusor" como algo distintivo de su profesión, papel que está atado a los valores de la objetividad, tal y como se define en los estudios de Johnston et al. (1976) y de Weaver y Wilhoit (1986 y 1996). Este hallazgo conversa, además, con los resultados de un análisis global comparativo realizado por Weaver (1996 y1999), en el que más de dos terceras partes de los doce países que midieron esta variable eligieron el rol de informador como "importante" o "bien importante". También, han llegado a la misma conclusión investigaciones realizadas en Argentina, España e Italia, entre otros (HUMANES, 1998; FAUNDES, 1998, p. 4; CANEL y SÁNCHEZ ARANDA, 1999, y MANCINI, 1999).

Sobre la función de informar, el 5 por ciento de los encuestados mencionó ese deber como lo único que distingue a la prensa - i.e., "necesidad de informar" y punto - pero, para muchos otros

\footnotetext{
${ }^{8}$ Estas contestaciones se distancian de cuando esta encuesta se hizo en Estados Unidos en 1999, en la que sobresalió la función democrática como el elemento más importante que distingue la profesión (Kovach y Rosenstiel, 2003: 27). En el presente estudio, la relación entre periodismo y democracia solo fue mencionada en un 6 por ciento.
}

Comum. \& Inf., v. 15, n.1, p. 105-131, jan./jun. 2012 
(44\%), se hizo necesario aclarar que la obligación de informar debe venir acompañada de otros valores y acciones importantes. Para el editor ejecutivo de un periódico, el compromiso de informar no debe circunscribirse al mero relato, sino que obliga al pensamiento y a derivar conocimiento

\begin{abstract}
Que informe asuntos o acontecimientos relevantes; que trascienda la mera información dando contexto; que investigue causas y efectos de los hechos, y los informe; que provoque análisis, que obligue al pensamiento crítico, que refleje un esfuerzo de balance y variedad de voces; y que, dejando atrás el rutinario "dijo", "puntualizó" y "agregó", desemboque en una propuesta de soluciones.
\end{abstract}

Este editor destaca que el contenido debe provocar reflexión y entendimiento, y que, como consecuencia, impacte a la sociedad, que construya un sistema mejor. En otras palabras, la labor del periodista no es la de "megáfono" o de "taquígrafo", que solo se circunscribe a los datos provistos por la fuente o se limita a la representación de falsos debates. Para este periodista, destaca el papel de interpretador, que señalaron las investigaciones de Weaver, Wilhoit y otros (1986, 1996, 2007 y BEAM et al., 2009), aunque ese atributo solo fue mencionado en un 15 por ciento en esta encuesta. Tal y como lo afirman Ortega y Humanes (2000, p. 205): “[I]nformar tiene un significado que rebasa ampliamente la simple narración de lo que acontece, para convertirse en referencia inexcusable para que cada persona construya su proyecto vital". O, como bien establecen Kovach y Rosenstiel (2003, p. 24), "lo que define los principios y el propósito del periodismo es algo más básico: la función que desempeña la información en la vida de todo ciudadano".

Para algunos periodistas entrevistados, la función de informar, también, debe estar guiada por un sentido ético, que respete la conciencia individual de sus profesionales, como dice una reportera de prensa escrita:

Hay muchos profesionales que comunican, pero el periodista se distingue porque tiene una ética particular y un compromiso con la información, la verdad y el balance que no necesariamente tienen otros trabajadores de la comunicación.

El compromiso de informar y de crear conocimiento no solo deben obligar al pensamiento y deben estar enmarcados en valores éticos, sino que, como afirman la reportera antes mencionada y otros, deben venir acompañados de la verdad (36\%). Esta mirada coincide con el pronunciamiento que hizo la Comisión Hutchins en 1947: "Ya no basta con reproducir los hechos verazmente. Ahora, es 
necesario informar la verdad que encierran los hechos". En otras palabras, la función de los periodistas es "averiguar los hechos y encontrarles un sentido" (Commission on the Freedom of the Press, $1947 \mathrm{y}$ Kovach y Rosenstiel, 2003, p. 60).

La información que se presenta debe ser de interés público, relevante y con datos veraces, según muchos periodistas encuestados. Mas, para lograr que la información esté apegada a la verdad, debe estar libre del poder, según un reportero de prensa escrita

El periodismo es el oficio de informar asuntos de interés publico [sic] a la comunidad. Para que el periodismo pueda ejercerse de forma adecuada, debe ser libre - con las menores presiones posibles y sin compromisos con el asunto a ser informado - y con datos veraces, claros y honestos.

Este periodista, al mencionar "con las menores presiones posibles", alude a que los valores periodísticos representan una búsqueda incesante de independencia. Debe ser responsabilidad del periodista buscar y llegar a ese valor. Como este reportero, Kovach y Ronsenstiel (2003, p. 61) explican:

Es, en realidad, de mayor ayuda, y más adecuado, entender la verdad periodística como un proceso - o un viaje prolongado - que comienza con el artículo de presentación de una noticia y se va construyendo a lo largo del tiempo.

Según el reportero antes citado, y otros pocos, para representar a la ciudadanía, la verdad debe servir de contrapeso a los sectores de poder. Como establece un editor de Internet, lo que diferencia al periodismo es "[e]l deber de comunicar oportunamente, críticamente y con veracidad los hechos que contraponen a la sociedad civil con el poder". Este periodista presenta el rol del "adversario del poder" como uno que deben asumir los profesionales de la información, aunque este reclamo solo encuentra eco en este estudio en un 6 por ciento.

No obstante, no solo se debe crear conocimiento con información relevante que obligue al pensamiento, formado con valores éticos, sino que, para reportar la verdad, el periodista debe ser capaz de distinguirla de la mentira, así como debe poder comunicarla de una forma clara y sencilla. Dice una periodista de prensa escrita: "El periodismo es tener la habilidad de poder discernir entre la verdad y la mentira, y comunicar eso de la manera más sencilla y clara posible.” O, como indica otra reportera de 
prensa escrita:

La primera característica en la lista debe ser la ética profesional, eso separa al periodista del chismoso. Le siguen el respeto y la búsqueda de la verdad, el apego a los datos y la inmediatez.

La búsqueda de la verdad se conjuga con la ética profesional, aunque ir tras la primera es en la práctica periodística un proceso inacabado; es una aspiración:

El periodismo intenta llegar a la verdad en un mundo confuso procurando discernir en primer lugar lo que es información fidedigna de todo lo que son informaciones erróneas, desinformación o información interesada, para luego dejar que la comunidad reaccione y el proceso de discernimiento continúe. La búsqueda de la verdad se convierte en un diálogo (KOVACH y ROSENSTIEL, 2003, p. 63).

En ese sentido, informar la verdad no solo debe estar libre del poder, sino debe servir para darles voz y oídos a los ciudadanos, otro de los valores fundamentales del periodismo mencionados por la Comisión Hutchins (1947) y Kovach y Rosenstiel (2003), pero apenas mencionado entre los encuestados (solo por dos periodistas, $2 \%$ ). Como señala una jefa de sección de prensa escrita sobre lo que distingue el periodismo:

Es el relato de lo que ocurre en la sociedad, contado desde sus diversos ángulos y cuyo mayor objetivo es tratar de comunicar la verdad. En ese sentido, el periodista debe ser el oído, la voz y el representante del pueblo y de sus intereses.

En esta postura coincide la directora de un noticiario televisivo sobre lo que distingue al periodismo: "La sed de que la verdad salga a flote y poder darles voz a aquellos sin voz".

A la función de informar y al valor de la verdad, como características que mayor número de periodistas mencionaron, le sigue reportar con objetividad, imparcialidad, justicia o balance (28\%). Expresa un reportero de televisión:

El periodismo es una profesión que persigue un ideal (no alcanzado) que consiste en llevar la información de la manera más objetiva y pura. Es informar de aquellos sucesos que afectan a diversos componentes de nuestra sociedad, con el compromiso inalcanzable de no ser manipulados.

Comum. \& Inf., v. 15, n.1, p. 105-131, jan./jun. 2012 
Para este periodista, la labor de la prensa debe guiarse por dos ideales: objetividad e independencia de la influencia de los sectores de poder, libre de manipulaciones. El reportero ve la objetividad como el escudo que protege la labor periodística de las presiones que provienen del gobierno y del mercado. Tal y como establece Tuchman (1999, p. 199): "La objetividad puede verse como ritual estratégico de protección para los periodistas ante los riesgos de su actividad profesional". Sin embargo, al adoptar los conceptos "pureza" y "objetividad", este periodista pone de lado su rol como interpretador. El reportero presume la información como si fuera aséptica, como si hablara por sí misma. Para este periodista, los datos se presentan y se definen ellos solos.

Se debe mencionar que un porcentaje muy bajo nombró dos atributos que se pueden clasificar en la categoría de activista-populista: entretener (1\%) e influir en la opinión pública (2\%). A todas luces, los periodistas entrevistados no perciben las características del activista-populista como distintivas de su profesión.

Cuando se les pide a los periodistas encuestados que expliquen en sus propias palabras los aspectos que distinguen la prensa, no existe una mayoría que coincida en los atributos específicos que singularizan su profesión. Mas, los que resaltan se relacionan mayormente con el impacto que la prensa tiene en la sociedad y con los valores que los guían en su quehacer periodístico. Dentro de estas dos categorías, los tres atributos que los periodistas mencionaron con mayor frecuencia fueron: el deber de informar, el apego a la verdad y la objetividad. Todas son características que responden al perfil del periodista "difusor", tal y como lo han definido Weaver, Wilhoit y otros (1986, 1996 y 2007). Ante este cuadro, la próxima sección mirará cuáles son los valores fundamentales de la profesión para los periodistas encuestados, ya cuando se les solicita que elijan entre las alternativas presentadas.

\section{6 ¿Valores fundamentales del periodismo?}

Si bien los periodistas en Puerto Rico no llegan a un consenso en cuanto a los atributos que distingue su profesión, cuando se les pide que escojan entre los valores fundamentales de la profesión enumerados por el Pew Research Center, tienden a seleccionar aquellos que descansan en sus rutinas profesionales enmarcadas en unos valores éticos en los que prima la percepción de su rol como “difusor”, más que en su independencia y responsabilidad social. El rol de "difusor" no solo distingue sino que constituye un valor fundamental de su profesión.

Comum. \& Inf., v. 15, n.1, p. 105-131, jan./jun. 2012 
Los periodistas encuestados identificaron como valores fundamentales de la profesión periodística: obtener los datos correctos (98.9\%), presentar las diversas perspectivas que conforman la historia periodística $(95.7 \%)$ y no publicar rumores (72\%). También, la mayoría piensa que proveer al menos dos fuentes para corroborar la información provista por recursos anónimos (68.8\%) y ser neutrales $(64.5 \%)$ son valores fundamentales de la profesión. No obstante, las opiniones se dividen en cuanto a otros dos valores: mantener la influencia de los dueños de los medios fuera de la cobertura (48.4\%) y hacer que la audiencia sea la obligación principal de su trabajo (47.3\%). Asimismo, un porcentaje bajo piensa que mantener distancia de las fuentes informativas (34.4\%) es uno de los valores fundamentales de la prensa. La tendencia de los resultados es la misma, independientemente del medio en que laboran o del tiempo que llevan en la profesión, no así el puesto que ocupan, como se verá más adelante.

Las rutinas metodológicas enmarcadas dentro de los valores éticos de la profesión — presentar los datos correctos, no publicar rumores, corroborar fuentes anónimas y mostrar las diversas perspectivas que componen la historia - son percibidas por los periodistas en Puerto Rico como fundamentos. Como establecen Ortega y Humanes (2000, p. 43)

Ante la ausencia de reglas culturales objetivadas y contrastadas en un campo específico, son las rutinas (que no son reglas de producir conocimiento, sino rituales interactivos) establecidas por los periodistas y su particular manera de percibir la realidad las que se erigen en principios incuestionables (son el equivalente funcional de los paradigmas científicos).

O sea, las rutinas del periodista "difusor", por su repetición, se convierten en las leyes científicas de la profesión.

La imparcialidad, el balance y la justicia también, como se vio en la sección anterior, no solo son atributos que muchos piensan distinguen al periodismo, sino que un 64.5 por ciento considera que "siempre ser neutral" es un valor fundamental de la profesión.

Estos datos conversan con el hallazgo de que cerca de ocho de cada diez (79.2\%). periodistas encuestados piensan que es posible ofrecer un relato verdadero y preciso de los eventos con el que todos puedan estar de acuerdo. Asimismo, en su mayoría, tienden a apoyar la premisa de que es posible que un periodista desarrolle un método sistemático para cubrir los eventos de una manera 
desinteresada, justa y equilibrada (61.2\%). Nuevamente, no se presentan diferencias significativas en los resultados por medio, experiencia laboral o puesto.

La postura de los periodistas ante el apego a la verdad se vincula con la concepción original que se tenía sobre la objetividad. La noción de que la labor periodística debe ser guiada por dicho valor surge en los Estados Unidos a principios del siglo XX, cuando se empezó a ver al periodista como un observador objetivo de la realidad, premisa que iba de la mano con el desarrollo de empresas periodísticas para un público masivo y con la creación de las agencias de noticias y del modelo informativo. Debe aclararse que el término "objetividad" en el periodismo de principios del siglo XX invocaba a la necesidad de que los periodistas elaborasen un método consistente de verificación de la información, "una forma de aproximarse a los hechos de modo transparente", que evitara que los aspectos culturales o personales interfirieran con la información provista. No se refería a que los periodistas "pudieran estar a salvo de las arbitrariedades o preferencias personales", a que la redacción que se adoptara fuera "objetiva" ni mucho menos a la pureza, higienizada, de la información. En otras palabras, a lo que se aspiraba era a que lo objetivo fuera el método de indagación y no el periodista. Pese a las confusiones que ha acarreado el término, la prensa moderna abrazó ese valor, y, con el tiempo, se ha sugerido que es el periodista quien debe ser objetivo. Esto ha llevado a muchos a rechazar la objetividad como un fundamento de la profesión periodística y a afirmar, como muchos periodistas, que esta es inalcanzable (KOVACH y ROSENSTIEL, 2003, p. 18, 102-105).

La postura ante el apego a la verdad, además, sugiere que algunos periodistas entienden su papel de intelectual aunque no se asuman como tales:

Este íntimo convencimiento de que su actividad desemboca en la generación de verdades, lleva al periodista a plantearse su profesión como una forma de "guía" o "faro" para su sociedad. Dos imágenes, por los demás, profusamente asociadas a los intelectuales (ORTEGA y HUMANES, 2000, p. 210).

Si bien se ven como difusores, apegados a la verdad, los periodistas encuestados ubican en un último plano la labor de interpretar la noticia (30\%), distinto a como se ha evidenciado en los estudios de Weaver, Wilhoit y otros (1986, 1996 y 2007), en los que los periodistas estadounidenses se perciben principalmente como interpretadores.

Los resultados de este estudio, sin embargo, cobran mayor significado cuando se examinan las 
contestaciones analizadas a la luz de los puestos que ocupan los periodistas en los medios encuestados. Aunque las opiniones de los periodistas se dividen en cuanto a los postulados que afirman que la audiencia debe ser su obligación principal y en cuanto a mantener a los dueños fuera de la cobertura, no es así cuando se examinan las posturas de los directores y editores. Cinco de los seis directores (83.3\%) y la mayoría de los editores $(65.2 \%)$ que contestaron esta encuesta piensan que mantener la influencia de los dueños fuera de la cobertura no representa un valor fundamental. Asimismo, solo dos de seis (33.3\%) directores ven a la audiencia como la obligación principal del periodista. No se debe perder de vista que tanto los directores como los editores trazan y ejecutan la política editorial y responden a la dirección del negocio periodístico. Estas posturas alimentan la cultura de la organización periodística, lo que incide en la forma en que los periodistas pueden percibir y, al fin y al cabo, ejecutar su rol.

Las opiniones de los directores sobre la lealtad hacia la audiencia chocan con uno de los valores enumerados por la Comisión Hutchins (1947) y Kovach y Rosenstiel (2003), quienes explican que el trabajo de los periodistas se debe a la ciudadanía. Los periodistas construyen una relación con su audiencia a base de los valores y del compromiso que tengan con la comunidad (KOVACH y ROSENSTIEL, 2003, p. 86). Es una relación que se basa en el bien común. De este modo, la audiencia representa la comunidad y hace visible — concretiza - el término ciudadanía. Nuevamente, estas percepciones llevan a cuestionarse cómo todas las posturas de los directores se traducen en las rutinas esbozadas por la organización periodística.

Para entender, en parte, el rechazo o ambivalencia ante el valor de mantener a los dueños alejados de la cobertura, se debe tomar en cuenta que en Puerto Rico varios propietarios de los medios ocupan posiciones directivas. Tal y como sucede en otros países, hoy día, "ninguna empresa se constituye al margen de la mentalidad profesional, entre otras razones porque cada vez son más los periodistas empresarios de medios de comunicación" (ORTEGA y HUMANES, 2000, p. 56-57). En la Isla, se puede identificar dicha práctica en varios medios informativos, particularmente en los de prensa escrita como El Nuevo Día, Primera Hora y El Vocero. Esto podría contribuir a la percepción de que mantener a los dueños fuera de la cobertura no debe considerarse un fundamento del periodismo, ya que son sujetos híbridos, empresarios-periodistas, y resulta difícil deslindar ambos papeles.

Los resultados antes presentados conversan, asimismo, con la denuncia de algunos periodistas quienes han señalado que las presiones empresariales inciden en la cobertura de los medios. Incluso, en 
esta encuesta, 71.6 por ciento de los periodistas piensan que los dueños influyen "mucho" (33.7\%) y "bastante" (37.9\%) en los temas que se cubren y cómo se cubren. Debe recordarse que, en algunos medios, los dueños tienen roles directivos, lo que puede contribuir a difuminar la línea distintiva entre periodistas y propietarios y, en consecuencia, ir naturalizando la injerencia de estos en el contenido periodístico.

Es significativo, además, que la mayoría de los periodistas encuestados no identifiquen mantener cierta distancia de las fuentes informativas como un valor fundamental del periodismo (34.4\%). La falta de elección de este valor resulta problemático, porque es la separación entre fuente y periodista lo que protege la labor de la prensa de posibles conflictos de interés así como le provee al periodista la libertad necesaria para reportar sin faltar a sus lealtades personales ni profesionales.

Entre los periodistas en Puerto Rico, no existe consenso sobre los atributos que distinguen su profesión, pero cuando se les da a escoger entre los valores fundamentales de la profesión, adoptan principalmente los que pertenecen al rol de “difusor". Además, se muestran ambivalentes hacia la lealtad que deben tener hacia la audiencia y a la independencia de que los dueños se inmiscuyan en la producción periodística, así como ponen de lado mantener la independencia de las fuentes. Mas, cuando se les pregunta directamente a los periodistas sobre el impacto de su trabajo en la ciudadanía y en un sistema democrático, sus posturas quedan más definidas.

\section{Servicio público, democracia y presiones comerciales}

Los periodistas — independientemente de su puesto, medio o experiencia laboral — tienden a coincidir en que el periodismo de hoy día es simultáneamente un servicio público y un sistema que protege la democracia. Sin embargo, las opiniones están divididas en cuanto a percibirlo como una empresa comercial que se debe a sus inversionistas como cualquier otro negocio.

Seis de cada diez periodistas perciben la prensa como un servicio público que provee información a la ciudadanía para que esta pueda gobernarse a sí misma (61.8\% piensa que es una frase que la describe "muy bien" o "bien") y siete de cada diez la ven como un medio que protege contra la corrupción y el abuso (67.8\% piensa que es una frase que la describe “muy bien” o "bien"). Incluso, cuando se les pregunta si la labor de los periodistas contribuye a que los políticos hagan bien su trabajo, un 85.4 por ciento de ellos coinciden con esta afirmación. En ese sentido, se presenta una aparente 
contradicción: ven al periodismo como un instrumento central de la democracia, aunque, como se vio en la sección anterior, las opiniones se dividen en cuanto a percibir, como un valor fundamental, que la audiencia sea su obligación principal.

Las opiniones también se dividen sobre la idea de que la prensa es una empresa que se debe a sus dueños como cualquier otro negocio (37.8\% piensa que es una afirmación que describe "bien " o “muy bien" al periodismo de hoy día y 36.9\% que es una afirmación que lo describe "mal "o "muy mal"). No obstante, para 57 por ciento de los periodistas que tienen entre ocho y doce años de experiencia, tal afirmación describe "mal" o "muy mal" el periodismo. Aquellos que se encuentran a mitad de camino en su carrera laboral se adhieren con mayor fuerza a la responsabilidad social de forma más evidente que el resto de los entrevistados. En ese sentido, los periodistas suelen enfatizar en el rol social que tiene la prensa, pero no en su rol económico. Los periodistas ven al periodismo como un servicio público alejado de los intereses comerciales

Los periodistas continúan percibiendo su actividad como un servicio público destinado a fines extraeconómicos. Es esta una importante razón para que perciban su trabajo como una práctica que trasciende ampliamente la esfera de los meros intereses económicos (ORTEGA Y HUMANES, 2000, p. 59-60).

Los periodistas en la Isla están divididos en cuanto a ver su oficio como un negocio. Por un lado, les cuesta percibir a los medios como una empresa más, aunque, por otro, no rechazan la injerencia de los propietarios en la creación de su producto, la noticia. Tampoco tienen problema alguno en aseverar el impacto social que tiene su labor, pese a que no pueden afirmar de forma mayoritaria, como atributos distintivos o valores fundamentales de su profesión, que la lealtad principal de su trabajo se deba a la audiencia y a declararlo independiente del poder y de las fuentes.

\section{Conclusiones}

En este estudio quedó plasmado que los periodistas en Puerto Rico perciben su rol principalmente como "difusores" de información, más que intérpretes o adversarios del poder. Abrazan la objetividad, la neutralidad y la verdad como los fundamentos de su profesión. Estos hallazgos se evidenciaron independientemente de los elementos organizativos valorados-medio en el que trabajan y puesto que ocupan - y de los años de experiencia.

Comum. \& Inf., v. 15, n.1, p. 105-131, jan./jun. 2012 
Estos resultados coinciden con otros estudios globales en los que los periodistas entienden que el rol de informador es crucial. No obstante, la primacía que los profesionales de la información en la Isla le otorgan al rol de difusor lleva a cuestionarse cómo estos enfrentan el campo periodístico en un periodo de transición que ha impactado tanto el contenido que producen como las mismas rutinas profesionales. Particularmente, resulta preocupante si se advierte que estos cambios están enmarcados en prácticas de extrema comercialización que han escalado, entre otras razones, por la competencia que se deriva de las plataformas ajenas a los medios informativos.

Si bien es cierto que, en un contexto de superabundancia informativa facilitada por la Internet, el que los periodistas en la Isla abracen el papel de difusores, apegados a la verdad, sirve para distinguir su trabajo del que se genera en otras plataformas no periodísticas. No es menos cierto que la adhesión principal al papel de difusor deja de lado otros roles más pluralistas que pueden definir y diferenciar su labor entre la marejada informativa que prolifera en las diferentes plataformas mediáticas. Particularmente, la interpretación no ocupa un lugar primordial para los periodistas encuestados, distinto a como lo perciben los profesionales de la información en los Estados Unidos. Aunque varios teóricos han establecido que es en la interpretación en donde reside el rol distintivo del periodista en una sociedad en la que prima la superabundancia informativa, otros precisamente ponen en duda que, en medio de una crisis económica, los periodistas puedan asumir el rol de interpretador. Los hallazgos de este estudio apuntan a esta última dirección.

Pese a la primacía del rol de periodista "difusor", los encuestados reconocen el impacto que tiene su profesión en la sociedad: protege contra la corrupción y fortalece el sistema democrático. No obstante, y como evidencia del terreno movedizo en el que se ubican, las opiniones se dividen sobre los valores de la profesión que afirman que la audiencia debe ser su lealtad principal y que la labor periodística debe ser independiente del poder de los dueños de la empresa, y colocan absolutamente de lado mantener distancia de las fuentes informativas. La primeras dos posturas fueron rechazadas por los directores encuestados, y, la segunda, por los editores, las cuales ponen en entredicho la autonomía profesional de los periodistas y la función de la prensa en un sistema democrático.

Varios estudios han demostrado que las condiciones organizativas inciden en la percepción que tienen los periodistas sobre los valores de su profesión. Algunos de los medios en Puerto Rico, particularmente los impresos, trabajan en núcleos corporativos familiares, cuyos propietarios, en 
ocasiones, se convierten en sujetos híbridos, empresarios-directores o dueños-periodistas, que influyen directamente en el contenido que producen, pero cuyos intereses están guiados también por el aspecto mercantil del negocio periodístico. Entonces, el día a día de algunos periodistas se ve influido por las determinaciones de los editores y de los dueños. No obstante, una cosa es que el trabajo del periodista se inserte en una empresa en la que se reconoce que los dueños influyen, y otra es que se muestren ambivalentes hacia el valor fundamental de mantener la influencia de los propietarios fuera de la cobertura. Quizás, porque la injerencia de los dueños se ha convertido en práctica común, para muchos periodistas representa un ejercicio artificial, y finalmente fútil, abrazar como un valor fundamental de la profesión el mantener a los dueños separados de la producción del contenido.

La ambivalencia que muestran los periodistas en la Isla hacia dejar de lado la influencia de los empresarios también conversa con la que exhiben cuando se les pregunta si su lealtad principal se debe a la ciudadanía. Si los periodistas no distinguen de forma mayoritaria que mantener la influencia de los dueños fuera de la cobertura es un valor del campo, ¿cómo se garantiza o se aspira a que el contenido que producen responda a la ciudadanía y no a los intereses del mercado? ¿Es posible que la lealtad hacia los propietarios, hacia su empresa, esté suplantando la lealtad que le deben a la audiencia? Si no tienen sus lealtades claras, ¿cómo podrán combatir la banalización informativa y las prácticas de extrema comercialización? Aunque está fuera del alcance de este ensayo identificar la influencia directa que tienen los dueños de los medios en las percepciones de los periodistas, sí es preocupante pensar que la injerencia de los directores-propietarios y las opiniones de los editores puedan ir naturalizando la creencia de que no hay que mantener a los dueños fuera de la cobertura o que su lealtad no se deba a la ciudadanía. La jefatura de los medios no es todopoderosa, pero establece unas líneas editoriales que delimitan o guían las acciones de los medios y de los sujetos que laboran en ellos. Las mismas prácticas organizativas van naturalizando la profesión periodística, van creándola, y van incidiendo en las formas de pensar de los periodistas.

Los resultados de este estudio se correlacionan con las advertencias que, ya desde la década de los cuarenta, hizo la Comisión Hutchins sobre cómo la influencia de los dueños puede ensombrecer las posturas políticas que sean contrarias a las de ellos y cómo la extrema comercialización puede torpedear el contenido periodístico responsable. Precisamente, esas preocupaciones que presentó la Comisión todavía figuran como problemas cuando se examina la percepción que tienen los periodistas 
en la Isla sobre los valores fundamentales de su profesión setenta años después. Los valores de la responsabilidad social entran en contradicciones con los intereses comerciales y con la forma en que pueden inmiscuirse en las rutinas de producción informativa.

Esta investigación lleva a reflexionar sobre si la adopción de los valores fundamentales del periodismo representa, hoy día, una utopía. En contextos de constricción económica —en los que permean prácticas de extrema comercialización y en los que se difumina con mayor celeridad la línea divisoria entre información y el aspecto comercial — habría que preguntarse cuánto espacio existe para calificar a la prensa como una "institución independiente, libre para vigilar a los demás poderes e instituciones de la sociedad", como reza uno de los valores fundamentales de la profesión periodística (KOVACH y ROSENSTIEL, 2003, p. 19 y 25).

Estos hallazgos, a todas luces, presentan un signo de alarma para el estado del periodismo en Puerto Rico en un periodo de crisis económica, cuyas prácticas se mueven con mayor fuerza hacia la extrema comercialización y que, como ya otros estudiosos han articulado, requiere que los periodistas asuman su rol de productores de conocimiento, de intelectuales, no de simples transmisores (ORTEGA Y HUMANES, 2000). Como plantean Kovach y Rosenstiel (2003, p. 19-20)

En el nuevo siglo, uno de los interrogantes más serios que puede plantearse la sociedad democrática es si la prensa independiente podrá sobrevivir. La repuesta dependerá de que los periodistas tengan la lucidez y convicción suficientes para determinar qué significa ser una prensa independiente, y de que al resto de los ciudadanos les importe tener una prensa independiente debido precisamente a su condición de ciudadanos.

La independencia relativa y la responsabilidad social representan valores fundamentales que podrían determinar la supervivencia del periodismo, y eso incluye pensar en la ciudadanía como la lealtad principal, poner freno a las presiones de los dueños y mantener distancia de las fuentes informativas. Al menos, los periodistas en la Isla creen que la prensa fortalece la democracia. Falta, entonces, que ese entendimiento teórico se traduzca en unas valoraciones y prácticas diarias. Como dice Hallin (2000, p. 233), son los fundamentos los que al fin y al cabo sirven como instrumentos de resistencia; es en nombre de ellos que los periodistas se pueden amparar para resistir o limitar (no erradicar) las manipulaciones que provienen de los sectores de poder políticos y comerciales. 
Ya pasada la primera década del siglo XXI, el que los periodistas en Puerto Rico pongan de lado la interpretación y la lealtad hacia la audiencia, entre otros valores, levanta serias interrogantes sobre el futuro de la calidad del contenido periodístico y, en consecuencia, la posibilidad de que el periodismo pueda servir de instrumento para fortalecer el sistema democrático en la Isla.

\section{Referencias}

BAGDIKIAN, Ben H. The Media Monopoly. Boston: Beacon Press, 1983.

BANNING, Stephen. The Professionalization of Journalism: A Nineteen Century Beginning. Journalism History 24 (4), 1998-99, p. 157-160.

BEAM, Randal A. Professionalism as an Organizational Concept. Trabajo presentado en la División "Communication Theory and Methodology" en la conferencia anual de la Association for Education in Journalism and Mass Communication, 2-5 de Julio, 1988.

BEAM, Randal A., David H. Weaver y Bonnie J. Brownlee (). Changes in Professionalism of U.S. Journalists in the Turbulent Twenty-First Century. Journalism and Mass Communication Quarterly, n. 86 (2), p. 277-298, 2009.

BIRKHEAD, Douglas. The Progressive Reform of Journalism: the Rise of Professionalism in the Press. En: HOGAN, J. Michael. Rethoric and Reform in the Progressive Era. v. 6. East Lansing: Michigan State University Press, 2003.

CANEL, María José y ARANDA, José Javier Sánchez. La influencia de las actitudes profesionales del periodista español en las noticias. Análisi, n. 23, p. 151-170, 1999.

Commission on the Freedom of the Press. A Free and Responsible Press.

A General Report on Mass Communication: newspapers, radio, motion pictures, magazines, and books. Illinois: The University of Chicago Press, 1974.

COSS, Luis Fernando. Análisis histórico de la noción del periodismo profesional en Puerto Rico: del siglo XIX al XX. Tesis doctoral. Departamento de Historia, Universidad de Puerto Rico, Río Piedras, 2007.

CUSTODIO COLlAZO, Marie. Sorprende la conexión. El Nuevo Día, año XL, 14 mayo 2010. p. 3435.

FAUNDES, Juan Jorge. Una perspectiva estratégica y compleja del periodismo latinoamericano. Diálogos de la Comunicación, 51. FELAFACS, Lima, 1998.

GUERRERO, Manuel Alejandro. Medios de comunicación y la función de transparencia. Cuadernos de transparencia. 3. ed. México: Instituto Federal de Acceso a la Información Pública, Centro de Atención a la Sociedad, 2008.

FRANCO, Guillermo. Efectos de las tecnologías digitales en el periodismo de América Latina y el Caribe: ¿Qué ha cambiado hasta ahora y qué transformaciones vienen?. Sala de Prensa, 129, agosto 2010, XI (6). Disponible en: <http://www.saladeprensa.org/>. Acceso en: 2 feb. 2011.

FULLER, Jack. What is Happening to News?: the information explosion and the crisis in journalism. Chicago: The University of Chicago Press, 2010.

HALLIN, Daniel C. Commercialism and Professionalism in the American News Media. En:

CURRAN, James y GUREVITCH Michael (Eds.). Mass Media and Society. 3. ed. Londres: Arnold, 
2000. p. 218-237.

HARCUP, Tony. Journalists and Ethics: the quest for a collective voice. Journalism Studies. 2002.3

(1): 101-114.

HERMAN, Edward S. y NOAM Chomsky. Manufacturing Consent: the political economy of the mass media. Nueva York: Pantheon Books. 1988.

HOYER, Svennik y Epp Lauk. The Paradoxes of the Journalistic Profession: An Historical Perspective. Nordicom Review, n. 24 (2), p. 3-18, 2003.

HUMANES, Ma Luisa. La profesión periodística en España. Revista Zer, Revista de Estudios de Comunicación, Universidad del País Vasco, 4 mayo 1998. Disponible en: <http://www.ehu.es/zer/ zer4/humanes12.html>. Acceso en: 12 ene. 2011.

IGARZA, Roberto. Nuevos medios: estrategias de convergencia. Buenos Aires: La Crujía Ediciones, 2008.

INTERNET usage statistics: the internet big picture world internet users and population stats. En:

Internet world stats: usage and population. Disponible en: <http://www.internetworldstats.com/stats. htm>. Acceso en: 7 oct. 2011.

JONES, Alex S. Losing the news: the future of the news that feeds democracy. Nueva York: Oxford University Press, 2009.

JOHNSTONE, John W., SLAWSKI, Edward J. y BOWMAN, William W. The news people: a sociological portrait of American journalists and Their Work. Urbana: University of Illinois Press, 1976.

KOVACH, Bill y Tom Rosenstiel. Los elementos del Periodismo. Traducido por Amado Diéguez Rodríguez. Madrid: Ediciones El País. 2003.

MANCINI, Paolo. La paradoja de los periodistas italianos: difusores teóricos, pero defensores prácticos. Problemas metodológicos e interpretativos. Comunicación y Sociedad, España, 1999. XII (2): p. 115-134.

MCCHESNEY, Robert W. The Problem of Journalism: a political economic contribution to an explanation of the crisis in contemporary US journalism. Journalism Studies, 2003. n. 4 (3): 299-329. MELLADO, Claudia. Periodismo en Latinoamérica: revisión histórica y propuesta de un modelo de análisis. Comunicar, 2009. XVII (13): 193-201.

MEYER, Philip. The Vanishing Newspaper: saving journalism in the information age. Columbia: University of Missouri Press, 2004.

NORDENSTRENG, Karle. Media Ethics in Europe: in Search of Core Values. En: PORTER; VINCENT (Eds.). Ethics and mass communication in Europe. Londres: University of Westminster: 2001, p. 27- 35.

ORTEGA, Félix y María Luisa Humanes. Algo más que periodistas: sociología de una profesión. Barcelona, Ariel Sociología, 2000.

PEW Project for Excellence in Journalism. The State of the News Media: An Annual Report on American Journalism. 2009. Disponible en: <http://www.stateofthemedia.org/2009/narrative_ overview_intro.php>. Acceso en: 24 feb. 2010.

PRITCHARD, David y MARC-FRANCOIS. Media Convergence and Changes in Quèbec Jounalists 'Professional Values. Canadian Journal of Communication. n. 35: 595-607. 2010. REESE, Stephen. Understanding the Global Journalist: A Hierarchy-of-influences Approach. Journalism Studies, 2001. n. 2 (2): 173-187.

SALAVERRÍA, Ramón y NEGREDO, Samuel. Periodismo integrado: convergencia de medios y 
reorganización de redacciones. Barcelona: Editorial Sol90, 2008.

SHOEMAKER, Pamela J. y Stephen D. Reese. Mediating the message: theories of influences on mass media content. 2. ed. Nueva York: Longman Publishers, 1996.

SIEGEL, Lee. El mundo a través de una pantalla: ser humano en la era de la multitud digital.

Barcelona: Tendencias Editores, 2008.

STEIMBERG, Oscar. Naturaleza y cultura en el ocaso (triunfal) del periodismo amarillo. CIC.

Cuadernos de Información y Comunicación 5, Universidad Complutense: 2000. p. 235-240.

TUCHMAN, Gaye. La objetividad como ritual estratégico: un análisis de las nociones de objetividad de los periodistas. Cuadernos de Información y Comunicación 4, Universidad Complutense, 1999. p. 199-217.

VALDETTARO, Sandra. Diarios: entre internet, la desconfianza y los árboles muertos. En: CARLÓN, Mario y SCOLARI, Carlos A. (Eds.). El fin de los medios masivos. Argentina: La Crujía: 2009. p. 4767.

WEAVER, David H. (Ed.). The global journalist: news people around the world. Nueva Jersey: Hampton Press, 1998.

WEAVER, David H. Las actitudes profesionales de los periodistas en un contexto global.

Comunicación y Sociedad, 1999. XII (2): 33-45.

WEAVER, David H. Journalists in comparative perspective: backgrounds and professionalism. The Public, $\mathrm{n}^{\circ}$ 3(4). 83-91, 1996.

WEAVER, David H. y WILHOIT, Cleveland. The American journalist: a portrait of U.S. news people and their work. Bloomington: Indiana University Press, 1986.

WEAVER, David H. y WILHOIT, Cleveland. The American Journalist in the 1990s: U.S. news people at the end of an era. Mahway, Nueva Jersey: Lawrence Erlbaum Associates, Publishers, 1996. WEAVER, David H. y BEAM, Randal A., BONNIE J. BROWNLEE, Paul S. VOAKES y G. y WILHOIT, Cleveland. The American Journalist in the 21st. Century. Mahwah, New Jersey: Lawrence Erlbaum Associates, Publishers, 2007. 